\title{
Comparison of large-scale solar magnetic feilds observed at the Sayan observatory with data of other observatories
}

\author{
Michael L. Demidov and Helen M. Golubeva
}

Institute of Solar-Terrestrial Physics, Siberian Branch of the Russian Academy of Sciences, Irkutsk, P.O.Box 4026, Russia: email: demid@iszf.irk.ru, golubeva@iszf.irk.ru

Comparison of solar magnetic fields observed with various telescopes and/or in different spectral lines is important to verify the data reliability from instrumental points of view, and to determine some physical processes responsible for the origin of polarized emission in the solar atmosphere with extremely complicated distribution of magnetic and thermodynamic parameters in an aperture of observation and along the line-of-sight. When compared observations use the same line, and data reveal a significant systematic discrepancies, the question arises about the reasons of that. If compared data are obtained in different spectral lines, the existence of differences between them could be related to peculiarities of the polarized emission formation in one or another line. In this case the question arises - data in which line are more reliable? This problem is especially acute with regard to observations in the FeI $\lambda 525.02 \mathrm{~nm}$ spectral line due to the big Lande factor $(g=3.0)$ and low excitational potentional of this line. Recently this problem has become especially urgent for some space weather tasks. Both the analysis of this problem and main bibliographic references can be found in [1].

The objective of this study is to compare the large-scale magnetic fields of the Sun measured at the Sayan observatory (SO) with data obtained at the J.Wilcox solar observatory (WSO) and at the Kitt Peak observatory (KP). Both the SO and the WSO use the FeI $\lambda 525.02 \mathrm{~nm}$ spectral line as a working one, while the KP uses the FeI $\lambda 868.9$ $\mathrm{nm}$ line $\left(g_{e f f}=1.667\right)$. In this paper we consider the full-disk magnetograms obtained during the period 01 April - 26 December. 2001. We selected for analysis 20 couples of the SO and WSO magnetograms and 27 couples of the SO and KP magnetograms. The time difference between observations not exceed 18 hours, but nevertheless it has appeared extremely important and was kept in mind using the differential rotation law of the Sun according [2].The analyzed data were registered with using different spatial resolutions: 175 " at the WSO, 1.15" at the KP, and 100" at the SO. To compare SO and WSO, SO data were interpolated onto given WSO coordinate grid (level 1) - 195 points on the solar disk. To compare SO and KP, KP data were averaged to get the SO resolution 349 points.

The results of the statistical analysis of the sampled data sets are presented in Fig.1.It is possible to see that the data are good enough correlated (the correlation coefficient $\rho$ is equal to 0.85 in the case of WSO-SO, and 0.82 in the case of KP-SO), but there are systematic discrepancies (the regression coefficient $R$ is equal to 0.75 in the WSO-SO case, and to 3.71 in the KP-SO case). It is interesting to notice that the spread of points grows, as the magnetic field strength increases.

The extremely interesting question is: are the magnetic strength ratios $R=B_{I} / B_{S O}$ (where $B_{I}$ are the field strengths measured at the WSO or KP observatories) distributed uniformly over the solar disk? Or are there some peculiarities? To this end, for the each combination of observatories the $R$ values for every pixel of magnetograms were 

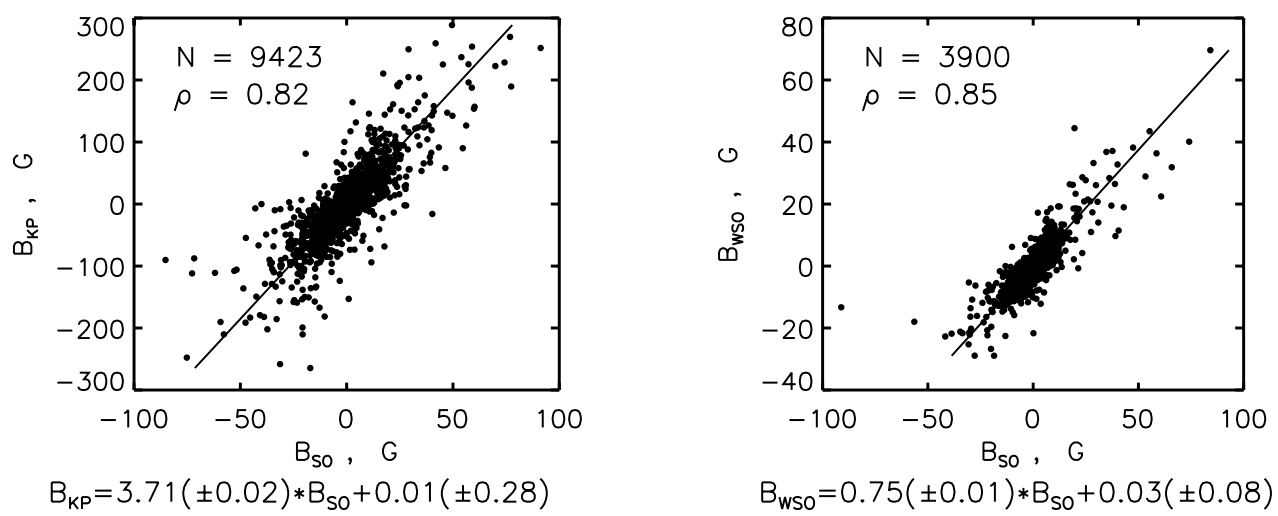

Figure 1. Comparison of the selected pairs of solar magnetograms obtained at the Sayn observatory and at the Kitt Peak (left panel) and J.Wilcox solar (right panel) observatories. $\mathrm{N}$ is the numbers of points, $\rho$ is the correlation coefficient. The corresponding linear regression equations are show below the graphics.
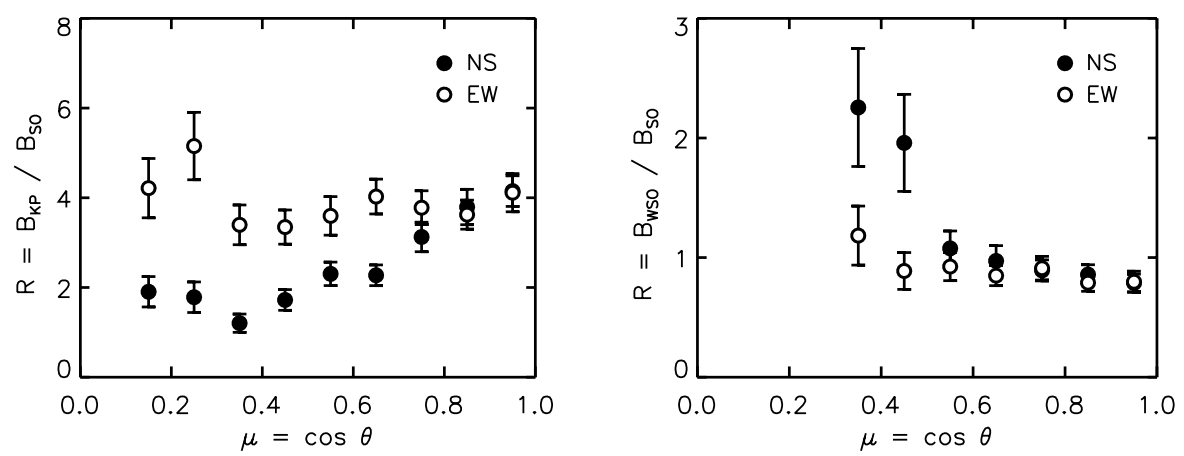

Figure 2. The center-to-limb-variations across the solar disk of the KP/SO (left panel) and WSO/SO (right panel) magnetic strength ratios $R$ for polar (NS) and equatorial (EW) sectors. estimated and the distributions of this parameter across the solar disc were calculated. A consideration of the results have shown that in the case of WSO-SO comparison the distribution of $R$ is enough homogeneous, except for the regions near the poles. In the case of the KP-SO comparison the distribution is noticeably in-homogeneous: the magnetic field strangths, measured at these two observatories, are compatible at the polar regions, but they show the appreciable discrepancy at the equatorial zone.

In order to study the polar-equator discrepancies in more detail, some additional estimation was performed and the results are shown in Fig.2 In the WSO-SO case the centre-to-limb variations (CLV) of $R$ for $\mathrm{N}-\mathrm{S}$ and $\mathrm{E}-\mathrm{W}$ sectors seem to be nearly identical. It should be waited for, because the same spectral line was used in both observatories. But there is except for the narrow near-limb zone. Concerning the KP-SO case, one can see that discrepancies in the CLV of $R$ for two sectors take place even at a short distance from the disc centre.

\section{Acknowledgements}

The results presented in this paper were obtained though partial support by the INTAS grant 2000-840 and RFBR grant 02-02-16467.

\section{References}

Demidov, M.L., Veretsky, R.M., \& Peshcherov, V.S. 2003 ASP Conference Series 307, 352-357. Howard, R., Harvey, J. 1970 Solar Phys. 12, 23-51. 